\title{
Appraisal in the diagnostic laboratory of three commercially available anaerobic cabinets
}

\author{
KD PHILLIPS, AT WILLIS \\ From the Public Health Laboratory, Luton \& Dunstable Hospital, Luton LU4 ODZ
}

SUMMARY Three commercially available anaerobic cabinets are described and their performance in relation to one another and to a standard anaerobic jar technique are reported upon from a clinical laboratory.

The successful isolation of anaerobes from clinical material is largely dependent on the use of a good anaerobic technique. Prereduced anaerobically sterilised (PRAS) media, ${ }^{1}$ and anaerobic cabinets ${ }^{2}$ have frequently been used to cultivate fastidious anaerobes, especially in studies concerned with the ecology of these organisms in the animal body. Although simple anaerobic jar techniques are entirely effective for the recovery of significant anaerobic bacteria from clinical material, ${ }^{34}$ it is appropriate that recently developed commercially available anaerobic cabinets should be considered for their application to clinical diagnostic bacteriology.

Three different commercially available anaerobic cabinets are considered here. These are the Forma Scientific 1024 Anaerobic Glove Cabinet (Raven Scientific Ltd, Haverhill, Suffolk), the Lab-Line 6500 PACE Anaerobic Chamber (Burkard Scientific (Sales) Ltd, Uxbridge, Middlesex), and the Whitley Anaerobic Cabinet (Don Whitley Scientific Ltd, Shipley, West Yorkshire). An examination of only one of each specification was reasonable, or indeed, possible. However, each example was among the first to be introduced commercially to the UK.

In the present short paper we describe these cabinets and report upon their performance in relation to one another and to the standard evacuation/replacement anaerobic jar technique used in this laboratory. ${ }^{5}$ Anaerobiosis in all three cabinets was achieved using gas mixtures of $10 \% \mathrm{H}_{2}, 10 \%$ $\mathrm{CO}_{2}$ and $80 \% \mathrm{~N}_{2}$ in the presence of palladium catalyst.

\section{Description of cabinets}

FORMA ANAEROBIC CABINET

The Forma cabinet is constructed in stainless steel

Accepted for publication 7 May 1981 with vinyl sleeves mounted in a flexible vinyl front panel. A rectangular interchange provides access to the chamber working area; air in the interchange is removed by automatic evacuation-gas purge sequences. The system is designed to maintain automatically a slight positive internal pressure; a sensor actuates the supply of mixed gas to the chamber when the pressure drops below a preset value. A forced draught incubator capable of holding up to 500 plates is incorporated in the system.

Provided the cabinet is regularly maintained, performs satisfactorily from a mechanical point of view, and a high degree of anaerobiosis was achieve - a weakly poised resazurin indicator was readily reduced. Condensation was a problem during periods of heavy usage, and when regular maintenance was not possible-for example, at holiday weekends. On these occasions it was often necessary to mop up the inside of the cabinet. Undue contamination of the interior surfaces by sporeforming organisms was not experienced, nor was contamination of culture plates a problem.

Worker discomfort due to perspiration associated with the prolonged use of rubber gloves was overcome by the use of thin cotton inner gloves or flock lined Marigold Industrial Supa-Weight gloves.

A regularly recurring problem with this equipment was the maintenance of the integrity of the inner and outer door gaskets; tight seals were difficult to obtain so that excessive mixed gas usage was common, leading to rapid exhaustion of the cylinder supply, and, incidentally, aggravating the condensation problem. The use of vacuum grease helped to eliminate leak problems but was both messy and not fully effective.

LAB-LINE ANAEROBIC CABINET

This instrument is very similar in concept and design to the Forma system although some advanced features are incorporated to its advantage. These 1110 
include a digital LED (light emitting diode) temperature/humidity display, alarm systems that indicate loss of positive pressure in the chamber and the incorrect sequence of operation of doors or switches, a gauge indicating positive pressure in the chamber, and an automatic lock on the outer door which prevents accidental opening when the interchange is in use. An attractive feature is the catalyst/ desiccant drawer through which the chamber atmosphere is circulated. This drawer can be removed from the cabinet from the exterior to allow changes of catalyst and desiccant. Curiously, the interchange is on the left side of the cabinet which is inconvenient for right-handed workers. The door gaskets are of excellent quality and provide a good seal leading to economy in the use of mixed gas. Mechanically, this instrument functioned satisfactorily and maintained a high degree of anaerobiosis as judged by resazurin reduction. The disadvantages inherent with the use of rubber gloves in the Lab-Line cabinet were similar to those outlined for the Forma cabinet.

\section{WHITLEY ANAEROBIC CABINET}

This British-designed cabinet which has recently become available incorporates a number of innovative features. The cabinet is constructed in stainless steel with a rigid polycarbonate front window; flexible vinyl panels are not employed. The cylindrical interchange is situated centrally in the roof of the cabinet and, as with the other cabinets, is microprocessor controlled. The cabinet is both a work station and an incubator, the whole working area being maintained at $37^{\circ} \mathrm{C}$. The interior cabinet temperature is shown by a liquid crystal display. A major advantage of this system is that the worker may use bare hands inside the cabinet. The entry ports have circular doors which are locked in position to seal the cabinet when the work station is not in use. Latex sleeves are provided which fit snugly and comfortably round the forearms. Air in these sleeves is evacuated from round the wrists, which become "vacuum packed", and replaced with mixed gas, these operations being controlled by foot switches. The operator can then unlock the entry port doors and work in the cabinet unencumbered by gloves. Small items can be introduced into the cabinet through the sleeves, thus reducing the use of the interchange. In addition to its internal catalyst sachet and "Anotox" wafer (see below) the cabinet has an external circulation system for the atmosphere which can be pumped through silica gel desiccant, additional catalyst, and additional atmospheric scrubber ("Anotox") which removes hydrogen sulphide and volatile fatty acids. Anotox is based on activated charcoal treated with ferric hydroxide, and its performance is reported upon elsewhere (JS Brazier, personal communication, 1981). This external "fail safe" circulatory system is actuated by a humidistat which controls the relative humidity in the chamber. The cabinet is economical of mixed gas despite the fact that nitrogen is not used in the interchange operation. A high degree of anaerobiosis is achieved in the cabinet and resazurin indicator is maintained in a reduced state. The cabinet is compact but roomy (holding up to 500 plates), and is very comfortable to work in.

\section{Cultural performance of cabinets}

The efficiency of the cabinets for the culture and isolation of obligate anaerobes was examined quantitatively and qualitatively; a wide variety of both stock laboratory strains of exacting anaerobic species including Bacteroides melaninogenicus and Clostridium haemolyticum, and fresh clinical specimens was used to make these assessments. These studies were carried out as described previously for anaerobic jars, ${ }^{67}$ being based essentially on comparative surface viable counts. ${ }^{8}$ The chemical indicator for anaerobiosis used was a weakly poised solution of resazurin. At that time, short of using a mass spectrometer, ${ }^{9}$ which was not available to us, there was no other practical alternative to enable a more exacting comparison of anaerobiosis to be made.

In general there were no overt qualitative or quantitative differences in performance between the three cabinets on the one hand and the standard anaerobic jar method of anaerobic culture on the other. In the case of clinical specimens, however, all the cabinets enabled earlier recovery of significant anaerobic isolates than was possible with anaerobic jars.

In all three cabinets plastic disposable loops were used because electric loop sterilisers were found to be unacceptably space-occupying and inefficient.

\section{Discussion}

Although all three anaerobic cabinets reviewed here produced and maintained an adequate anaerobic atmosphere, we found that the Lab-Line cabinet is marginally superior in design to the Forma cabinet, and the Whitley cabinet is clearly superior in design, operation and versatility to both of the others. The prices of these cabinets are comparable. It should be obvious that a comparison of complex equipment, based on one example of each cannot necessarily predict the likely performance of identical massproduced items. This is as true for anaerobic cabinets as it is for cars! 
In the operation of all anaerobic cabinets it is important to economise on gas usage because the "special" mixed gases required are very expensive. In this connection a common problem that may occur is inadequate integrity of the inner and outer door gaskets; unless tight seals are obtained, grossly excessive mixed gas usage will occur, leading to rapid exhaustion of the cylinders; this is not only wasteful and exceedingly expensive, but may lead to failure of anaerobiosis due to lack of gas, and also to the accumulation of large excesses of condensate. An obvious heavy demand for gas occurs when the entry interchange is opened frequently. One of the great advantages of the Whitley cabinet is that small items can be introduced into or removed from the cabinet without passing through the air lock. Gas leaks may develop at a great variety of points in this type of complex equipment, and they may be very difficult to trace; a highly sensitive portable gas leak detector is provided by Don Whitley Ltd, and we regard it as essential for users of anaerobic cabinets.

We found the single mixed gas cylinder employed on the Whitley cabinet more convenient than the use of both a mixed gas cylinder and a nitrogen cylinder (for air lock purging), which are employed by the other systems.

In any anaerobic environment produced by hydrogen/oxygen catalysis, excessive humidity is likely to cause problems. This is especially the case in anaerobic cabinets in which water condensate is derived, not only from "continuous" catalysis, but also from a large bulk of constantly changing media. This problem has been overcome in the Whitley cabinet by the incorporation of a humidistat which can be set between $20 \%$ and $90 \%$. The humidistat controls the operation of a "fail safe" external circulating system through which the atmosphere of the cabinet is pumped. Incorporation of an atmospheric scrubber into this unit marks a notable advance in cabinet design since it not only prolongs the life of the palladium catalyst but also enhances bacterial growth by removing toxic metabolic products from the atmosphere.

The Forma and Lab-Line cabinets are both provided with an internal incubator, separated from the actual working area which is slightly above ambient temperature. The Whitley cabinet, on the other hand, is both an incubator and a work station, a benefit made more significant by the fact that gloves are not employed. This in turn permits unimpaired manual dexterity and complete comfort during long periods of work.

In the past, anaerobic cabinets have been employed chiefly as research tools, and specialist workers in the field have usually designed and constructed their own systems with a considerable degree of success. This specialist aspect may have precluded the adoption of the technique in laboratories which could have benefited from the use of an anaerobic cabinet in conjunction with, or as an alternative to, their conventional anaerobic methods. Considerable practical advantages may be obtained from a combination of the methods although it should be borne in mind that carefully used, modern anaerobic jars yield isolations rates of clinically significant anaerobes which are comparable to those obtained in anaerobic cabinets. Selective use may be made of the anaerobic cabinet for specimens suspected of containing anaerobes, and cultures can be inspected frequently without the disadvantage of exposure to air or the temperature drop associated with examination of anaerobic jar cultures; these were doubtless important factors in the earlier recognition of anaerobic growth. Prolonged incubation of cultures is facilitated by the cabinet and may reveal an unsuspected anaerobic bacterial presence in specimens previously regarded as either sterile or containing no significant bacteria. Moreover, cabinets permit the examination of cultures at frequent intervals throughout the day (and night).

A well designed and regularly maintained anaerobic cabinet thus offers both the diagnostio and research laboratory a continuity of anaerobiosis which is intrinsically more efficient than the cyclifo operation of multiple anaerobic jars. The use of commercially available anaerobic cabinets will undoubtedly become more widespread, particularly if their cost effectiveness rises relative to anaerobic jar methodologies.

Despite these comments it is important to recognise that anaerobic cabinets are not a substitute for poorly performed conventional anaerobic techniques; that those who fail with anaerobic jars will also fail with cabinets. Moreover, for diagnostic laboratories whose anaerobic commitment is small (say, less than 15 jars per day), commercial anaerobic cabinets are not cost-effective compared with an efficient anaerobic jar procedure.

We thank Mr P Tearle, Mrs J King, Mr J Brazier and Mrs G Gottobed for technical assistance and Mrs M Senjack for the typing of this manuscript.

\section{References}

${ }^{1}$ Moore WEC. Techniques for routine fastidious anaerobes. J Systemat Bact 1966;16:173-90.

${ }^{2}$ Aranki A, Syed SA, Kenny EB, Freter R. Isolation by anaerobic bacteria from human gingivia and mouse cecum by means of a simplified glove box procedure. Appl Microbiol 1969;17:568-76.

${ }^{3}$ Killgore GE, Starr SE, Del Bene VE, Whaley DN, Dowell, 
VR. Comparison of three anaerobic systems for the isolation of anaerobic bacteria from clinical specimens. Am J Clin Pathol 1973;59:552-9.

4 Watt B, Collee JG, Brown R. The isolation of strict anaerobes: the use of an anaerobic cabinet compared with a conventional procedure. J Med Microbiol 1974; 7:315-24.

${ }^{5}$ Willis AT. Anaerobic bacteriology: clinical and laboratory practice 3rd ed. London; Butterworths, 1977.

${ }^{6}$ Ferguson IR, Phillips KD, Willis AT. An evaluation of the "Gas Kit" disposable hydrogen and carbon dioxide generator for the culture of anaerobic bacteria. J Appl Bacteriol 1976;41:433-7.
7 Burt R, Phillips KD. A new anaerobic jar. J Clin Pathol 1977;30: 1082-4.

${ }^{8}$ Miles AA, Misra SS. The estimation of the bactericidal power of blood. J Hyg (Lond) 1938;38:732-48.

- Tabaqchali S, Peach S, Sneddon W. Mass spectral measurement of residual oxygen in the atmosphere of an anaerobic culture chamber. J Clin Microbiol 1978;8: 339-41.

Requests for reprints to: Mr KD Phillips, Public Health Laboratory, Luton and Dunstable Hospital, Luton LU4 ODZ, England. 